\title{
Measurement of neoclassically predicted edge current density at ASDEX Upgrade
}

\author{
M.G. Dunne ${ }^{1}$,P.J. McCarthy ${ }^{1}$, E. Wolfrum ${ }^{2}$, R. Fischer ${ }^{2}$, L. \\ Giannone $^{2}$, A. Burckhart ${ }^{2}$, ASDEX Upgrade Team ${ }^{2}$ \\ ${ }^{1}$ Department of Physics, University College Cork, Association Euratom-DCU, Cork, \\ Ireland \\ ${ }^{2}$ Max-Planck-Institut für Plasmaphysik, EURATOM Association, D-85748 Garching, \\ Germany \\ E-mail: michael.dunne@mars.ucc.ie
}

\begin{abstract}
Experimental confirmation of neoclassically predicted edge current density in an ELMy H-mode plasma is presented. Current density analysis using the CLISTE equilibrium code is outlined and the rationale for accuracy of the reconstructions is explained. Sample profiles and time traces from analysis of data at ASDEX Upgrade are presented. A high time resolution is possible due to the use of an ELM-synchronisation technique. Additionally, the flux surface averaged current density is calculated using a neoclassical approach. Results from these two separate methods are then compared and are found to validate the theoretical formula. Finally, several discharges are compared as part of a fuelling study, showing that the size and width of the edge current density peak at the low field side can be explained by the electron density and temperature drives and their respective collisionality modifications.
\end{abstract}




\section{Introduction}

Accurate knowledge of the current density at the edge of the plasma is important for analysis of the instabilities it contributes to. One such instability is the edge localised mode (ELM). The currently most favoured theory to describe ELMs is the peeling-ballooning theory[1]. According to this theory, type-I ELMs can be analysed by considering the pressure gradient and current density at the edge of the plasma. Type-I ELMs are modelled as reaching a pressure gradient limit, followed by an edge current density limit.

Since the Type-I ELMy H-mode is the preferred operating scenario for ITER, it is desirable to understand the physics of these events and how best to control them. ELM crashes occur over a very short timescale $(\approx 50 \mu \mathrm{s})$ and can result in the release of up to $10 \%$ of the plasma stored energy in a short time[2]. This poses problems for the lifetime of the plasma facing components in a reactor size tokamak such as ITER [3, 4].

ASDEX Upgrade, a medium sized tokamak, has highly temporally and spatially resolved diagnostics which allow the electron temperature and density [5, 6], and hence pressure, profiles to be accurately determined. It has been postulated that while the pressure gradient remains at the critical value for some time, the current density may take longer to build up due to the resistive time of the plasma. A theoretical estimate in [6] has shown that this is unlikely. A more complete analysis would include a measurement of the current density.

Direct measurements of the edge current density in a tokamak can be achieved by using a Motional Stark Effect (MSE) diagnostic, such as at MAST[7]. A second option is to measure the Zeeman splitting of lithium $2 \mathrm{~s}-2 \mathrm{p}$ transition lines, such as at DIII-D[8]. At ASDEX Upgrade, neither of these options are currently available. These measurements are also very challenging due to the very small effect of a large current peak on the flat poloidal field profile at the plasma edge; the unique conditions presented in a spherical tokamak lessen the impact of this particular issue, allowing the MAST measurements to be made accurately.

Contributions to the local current density in the confined edge region of a tokamak plasma were considered from three main sources: Ohmic current, Pfirsch-Schlüter current, and bootstrap current[9]. Fast ions, while present in the core of the plasma, were assumed to have a negligible impact on both the pressure and current drive in the pedestal region. This will be further explained in section 2.2. Accurately calculating the Ohmic, Pfirsch-Schlüter, and bootstrap contributions requires knowledge of the local plasma magnetic geometry as well as temperature and density data.

A general formula to describe the bootstrap current, referred to here as $j_{\text {boot }}$, for arbitrary collisionality has been derived by Sauter et al.[10, 11]. It is dependent on temperature and density gradients in the plasma and is given by

$$
\left\langle\boldsymbol{j}_{\text {boot }} \cdot \boldsymbol{B}\right\rangle=F(\psi) p(\psi)\left[\mathcal{L}_{31} \frac{\partial \ln n_{e}}{\partial \psi}\right.
$$




$$
\begin{aligned}
& +R_{p e}\left(\mathcal{L}_{31}+\mathcal{L}_{32}\right) \frac{\partial \ln T_{e}}{\partial \psi} \\
& \left.+\left(1-R_{p e}\right) \times\left(1+\frac{\mathcal{L}_{34}}{\mathcal{L}_{31}} \alpha\right) \mathcal{L}_{31} \frac{\partial \ln T_{i}}{\partial \psi}\right]
\end{aligned}
$$

where $\mathcal{L}_{31}, \mathcal{L}_{32}$, and $\mathcal{L}_{34}$ are the collisionality dependent coefficients given in $[10,11]$. $R_{p e}$ is the ratio of electron pressure to total pressure (typically $1 / 2$ ), and $\alpha$ is also related to the collisionality. This neoclassical approach has previously been validated by Wade et al.[12] by using a starting equilibrium and a current diffusion model to determine the electric field and compare with the neoclassical prediction. This was further validated by Kelliher et al.[13] and compared to MSE measurements at JET. Here, we use a different method for the comparison. We calculate the neoclassical flux surface averaged current density defined as

$$
\left\langle\boldsymbol{j}_{\text {neo }} \cdot \boldsymbol{B}\right\rangle=\left\langle\boldsymbol{j}_{\text {boot }} \cdot \boldsymbol{B}\right\rangle+\left\langle\boldsymbol{j}_{\text {Ohmic }} \cdot \boldsymbol{B}\right\rangle
$$

using the input kinetic profiles and the flux functions $F(\psi), p(\psi)$ from CLISTE and compare this value to $\langle\boldsymbol{j} \cdot \boldsymbol{B}\rangle$ from CLISTE, which is given by

$$
\langle\boldsymbol{j} \cdot \boldsymbol{B}\rangle(\psi)=F(\psi) p^{\prime}(\psi)+F^{\prime}(\psi) \frac{\left\langle B^{2}\right\rangle}{\mu_{0}}
$$

where $p^{\prime}(\psi)$ and $F F^{\prime}(\psi)$ are the source profiles for the Grad-Shafranov equation (see section 2). $\left\langle\boldsymbol{j}_{\text {boot }} \cdot \boldsymbol{B}\right\rangle$ is calculated from equation 1. $\left\langle\boldsymbol{j}_{\boldsymbol{O h m i c}} \cdot \boldsymbol{B}\right\rangle$ is calculated using neoclassical resistivity and current diffusion.

\section{Reconstruction method and input data}

The measurement for determining the current density employed in this work exploits an equilibrium solver. The local current density is a direct output of the Grad-Shafranov equation, given by

$$
-\Delta^{*} \psi=\mu_{0} R^{2} \frac{d p}{d \psi}+F \frac{d F}{d \psi}=\mu_{0} R j_{\phi}
$$

Use of the CLISTE code[14, 15], which has the ability to model current into the plasma scrape off layer (SOL), allows an accurate reconstruction of the current density. This can then be compared to $\left\langle\boldsymbol{j}_{\text {neo }} \cdot \boldsymbol{B}\right\rangle$ independently calculated from equation 2 .

\subsection{Magnetic and current measurements}

In order to produce an equilibrium, several inputs in the form of fitted profiles are required. To improve the quality of this data set, all signals were synchronised to an ELM and averaged in time to achieve a $100 \mu$ s time resolution. An example of this averaging is shown for the total plasma current in figure 1. The rise of the plasma current shown corresponds to the onset time of the ELM. However, with a typical time resolution of $1 \mathrm{~ms}$ this is not used as a synchronisation signal; instead, the outer divertor current, shown in figure 2 , is used. 


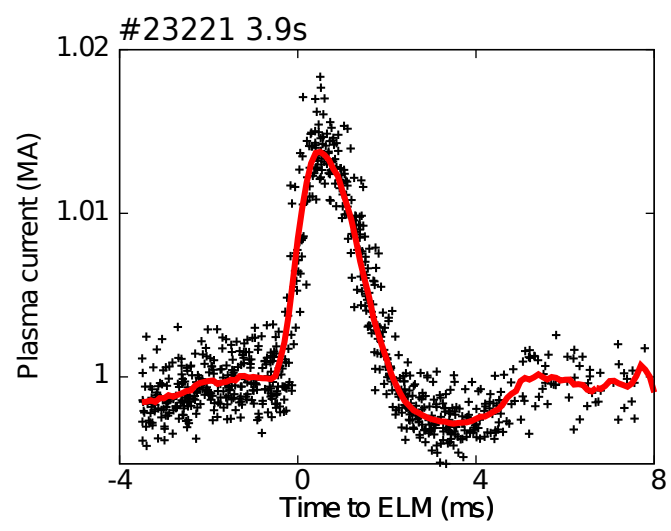

Figure 1. (Colour online) Total plasma current synchronised to an ELM. The increase is most likely due to an overcompensated inductive response to the loss of edge current density.

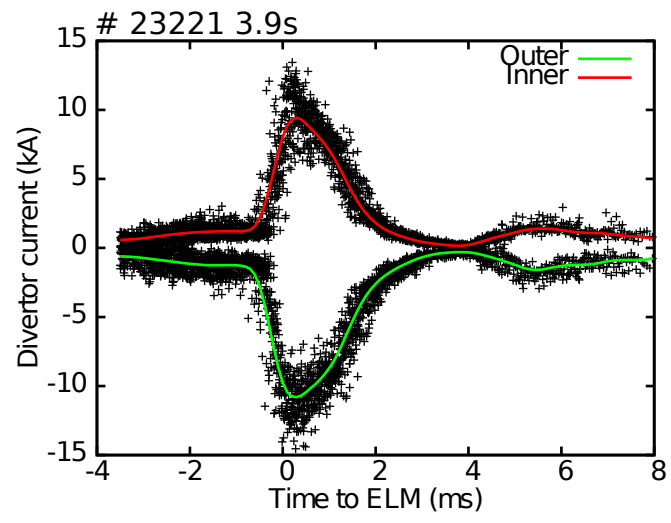

Figure 2. (Colour online) ELM synchronised divertor currents. The currents presented here are measured using a shunt resistance with the convention that positive current flows into the divertor tile. Thus, the current flows from the outboard tiles, along the field lines and into the inboard tiles.

At ASDEX Upgrade approximately 60 magnetic signals are routinely used to calculate equilibria in both real time and on an inter-shot basis. These diagnostics act as the principal constraints on an equilibrium reconstruction; any reaction these show to the ELM and the plasma recovery will have a large impact on the output current profile evolution. Standard equilibrium magnetic measurements have recently been shown to contain useful information to identify the edge current profile[15].

Typically, currents in the scrape off layer at ASDEX Upgrade are composed of Pfirsch-Schlüter and Ohmic drives, and thermoelectric currents[16]. The direction of these currents is into the divertor at the high field side, through the structure and out at the low field side. These poloidal currents, shown in figure 2, act as constraints for the SOL current. Each tile current measurement specifies the difference in the flux function $F(\psi)=\mu_{0} \frac{I_{p o l}(\psi)}{2 \pi}$ across the tile. The high time resolution of $0.01 \mathrm{~ms}$ also allows them to be used as an ELM indicator signal to which all other data can be synchronised. All 
data in this paper were synchronised to the times of peak divertor current, i.e. the time of peak particle loss due to the ELM crash.

\subsection{Pressure measurements}

The CLISTE equilibrium solution is also constrained by an edge pressure profile created using the same synchronisation shown above, with temperature and density data taken from Integrated Data Analysis (IDA)[17]. IDA is a Bayesian approach to data analysis used to generate temperature and density profiles by combining complementary diagnostics. Electron temperature input for this analysis was from an electron cyclotron emission (ECE) diagnostic[18] and electron density from a combination of Lithium beam[19, 20] and DCN-interferometry[21]. The ECE diagnostic has a sampling rate of $31 \mathrm{kHz}$ and a spatial resolution of $1 \mathrm{~cm}$, while the edge Lithium beam has a sampling rate of $20 \mathrm{kHz}$ and a spatial resolution of $5 \mathrm{~mm}[5]$. The high resolution of these diagnostics allows the pedestal structure (of the order of $2 \mathrm{~cm}$ in ASDEX Upgrade) to be determined. Ion temperatures were taken to be equal to electron temperatures, as shown for this case by Wolfrum et al.[5], and the ion density to be 0.85 that of the electron density, as derived from a typical value for $Z_{e f f} \approx 1.8$ and the main impurities being Boron and Oxygen.

One of the assumptions made in this analysis is that the contribution of fast ions to equilibrium force balance in the plasma edge is negligible. The fast ion pressure profile was calculated with the FAFNER code[22] and verified that the contribution of the fast ions is of the order of a few percent at the pedestal top and effectively vanishes in the pedestal. In the core of the plasma, the fast ion pressure become more prominent; in this case $\approx 30 \%$ of the total core pressure is due to fast ions. While it is possible to include the fast ion pressure profile in CLISTE and thus constrain the entire equilibrium pressure profile, this was not done in the present analysis since the focus was principally on the edge region. Accordingly, thermal pressure measurements, although available over the full plasma radius, were used to constrain the equilibrium pressure in the edge region only.

\section{Current density profiles}

Results presented in this section are from ASDEX Upgrade discharge \#23221, which is a Type-I ELMy H-mode discharge, having 7.5 MW of Neutral Beam Injection heating, 1.3 MW of Electron Cyclotron Resonance Heating, a 1 MA plasma current, -2.5 T toroidal magnetic field and $5.8 \times 10^{21} \mathrm{~s}^{-1}$ Deuterium gas injection. Data was analysed between 3.6 and 4.4 seconds of the discharge.

Figure 3 shows an example of the difference the pressure constraint can make to the edge current density as calculated in CLISTE. The two profiles presented were created with the same curvature constraints, the red with and blue without an input pressure profile. The boxes show the input data points. The effect of the divertor currents can be clearly seen in the SOL region of the current density fit where the error bars are much 


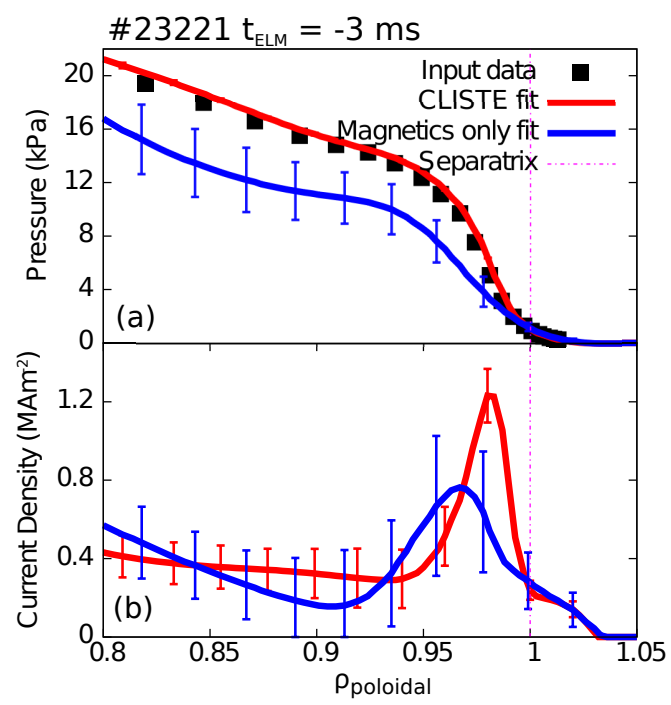

Figure 3. (Colour online) (a) Pressure profile. (b) current density profile. The blue line denotes a fit made only with magnetic and divertor current constraints, while the red line is the fit using an additionally prescribed pressure profile. Black boxes represent input data points. Error bars are 1 sigma confidence bands.

smaller than inside the separatrix and both profiles agree closely. Since these currents are measurements of the poloidal SOL current, this provides an integral constraint of the ff' source profile on and outside the separatrix.

Once we move away from the SOL, the two current density fits begin to diverge, and the error bars increase substantially in the magnetics-only case. The error bars shown here (and in all subsequent plots) are one sigma confidence bands, as calculated by CLISTE, and described in section 3.1. The flatter current density profile in the magnetics-only case is due to the internal curvature constraint in CLISTE, which aims to minimise the value and curvature of the knots in the profile spline fit.

\subsection{Confidence band calculation}

The starting point for calculation of error bars for individual scalar parameters and confidence bands for plasma profiles is the $\mathrm{NxN}$ variance-covariance matrix $\mathrm{V}$ of the $\mathrm{N}$ free spline coefficients that parameterize the p' and ff' source profiles and hence the current density profile. This matrix is returned by the linear regression routine that optimizes the fit to the data at each iteration cycle. The diagonal of this matrix holds the variances, i.e. squared standard deviations, for the fitted parameters, while the off diagonal terms hold the covariances.

If $\mathrm{p}$ is any parameter of the interpreted equilibrium and $\nabla_{\alpha} p$ is the gradient vector of $\mathrm{p}$ with respect to the set of fitted parameters $\alpha_{i}$, then $\sigma(p)$, the standard deviation for $\mathrm{p}$, is given by:

$$
\sigma^{2}(p)=\left(\nabla_{\alpha} p\right)^{\top} \cdot V \cdot \nabla_{\alpha} p
$$


Confidence bands for spatial profiles (e.g. as a function of major radius R) can be constructed in a pointwise manner by treating each element of a regularly spaced array of profile values as a separate parameter and interpolating the calculated array of standard deviations to form a continuous function of $\mathrm{R}$.

Equilibrium reconstruction is an ill-conditioned inverse problem and the error bars obtained by the above procedure are specific to the choice of current profile parameterisation and the choice of regularisation penalty. Tikhonov-type regularisation is used here, where the magnitude of the spline coefficients and the curvature at each knot location are penalised. The choice of the regularisation parameter is guided by methods such as the L-curve and Morozov's discrepancy principle [23].

In the case of the current density, the magnitude of the confidence bands is calculated from the covariances with respect to the ff' and p' source profiles, since j depends on both of these. In the magnetics-only case, both source profiles are internally free and constrained only by external information; in the kinetically constrained case the p' profile in internally constrained in the pedestal region, lowering the contribution of these coefficients to the confidence band calculation.

\subsection{Time dependence of peak edge current density}

Time traces of the peak pressure gradient and the low field side peak edge current density, for kinetic and non-kinetic constraints, are shown in figure 4. A sharp drop in the edge current density is observed, similar to that of the pressure gradient. The current density then recovers on a similar timescale as the pressure gradient. This finding, coupled with the constant pre-ELM current density and consistent with the model in [6], is therefore inconsistent with the theory of a resistive delay in the current density growth being responsible for the ELM crash.

The rapid drop of the edge current density at the ELM crash initially seems somewhat surprising; one would expect a decrease (and subsequent increase) in line with a resistive delay relative to the pressure gradient collapse. However, this becomes easier to understand when one sees that the plasma circumference shrinks rapidly at the ELM crash, as shown in figure 5. This corresponds to a movement of the inner and outer separatrix location of approximately $5 \mathrm{~mm}$ in each case, shown in figure 6. This reduction in the size of the plasma could lead to a large portion of the edge region (the pedestal being $\approx 20 \mathrm{~mm}$ wide) being removed to the SOL, allowing the plasma to lose current faster than expected. Support for this hypothesis is given by a larger movement of the inner separatrix location, which, due to the larger flux expansion, implies that a fixed amount of flux is separated from the entire plasma surface. Results in figure 6 (and all further plots) are from the CLISTE results constrained with magnetic, kinetic and divertor current data. It can also been seen in figure 6 that the inward motion of the magnetic axis is delayed by $\approx 2-3 \mathrm{~ms}$ with respect to the separatrix contraction. This inward movement after the ELM crash is expected due to the loss of pressure, and the delay corresponds to the pedestal resistive timescale of $\approx 1.5 \mathrm{~ms}$; this 


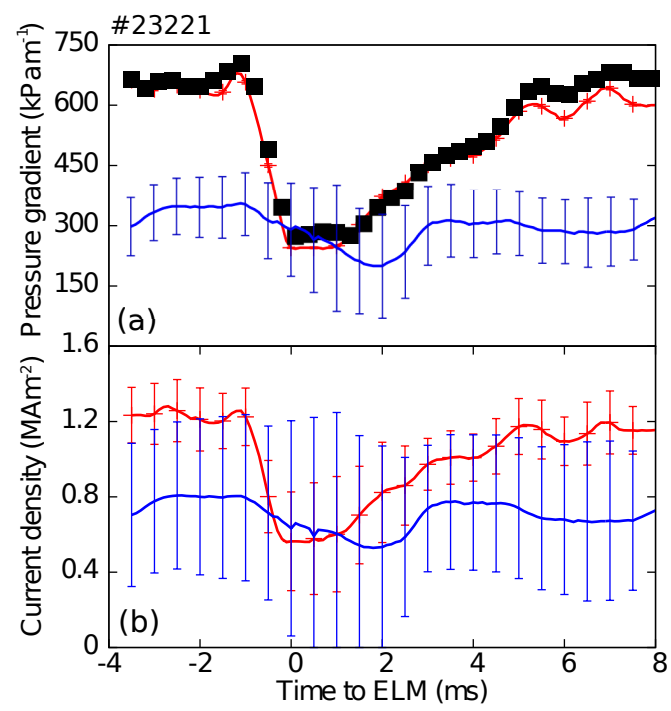

Figure 4. (Colour online) (a) Maximum pressure gradient time trace. (b) peak edge current density time trace. The colours indicate the different constraints, as in figure 3. The current density closely follows the evolution of the pressure gradient in both cases.

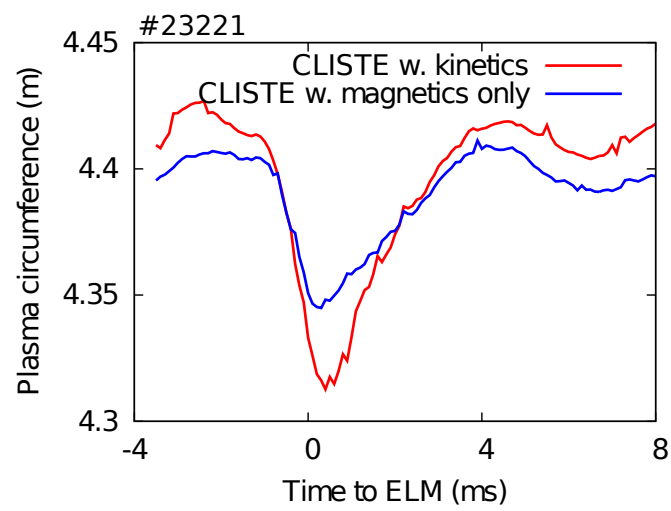

Figure 5. (Colour online) Time trace of plasma circumference starting just prior to $\mathrm{t}=0$. Shown in red is the circumference corresponding to the kinetically constrained CLISTE, while the blue shows CLISTE with magnetics measurements only. Both show a robust drop in the circumference, corresponding to a $5 \mathrm{~mm}$ movement of the outer and inner separatrix locations towards the plasma centre.

was determined using an average pedestal resistivity of $3 \times 10^{-6} \Omega \mathrm{m}$ (calculated from neoclassical resistivity) and a pedestal width of $2 \mathrm{~cm}$.

\section{Comparison with neoclassical current density}

In order to make a comparison between the CLISTE output and $j_{\text {neo }}$, the flux surface averaged current density was analysed. The calculation of $j_{\text {neo }}$ results in a value of $\left\langle\boldsymbol{j}_{\text {neo }} \cdot \boldsymbol{B}\right\rangle$, which can be compared to $\langle\boldsymbol{j} \cdot \boldsymbol{B}\rangle$ from the CLISTE code. By using these 


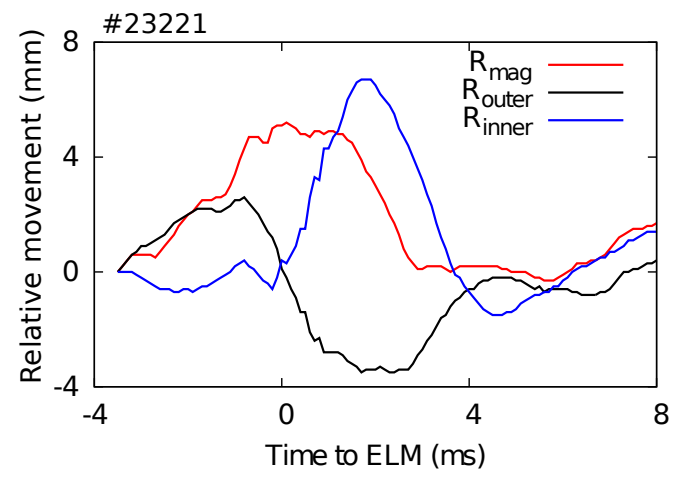

Figure 6. (Colour online) Time traces of movement of magnetic axis (red), outer midplane separatrix location (black) and inner midplane separatrix location (blue). All traces are taken relative to the first timepoint at $3.5 \mathrm{~ms}$ prior to the ELM crash.

values, the Pfirsch-Schlüter current is also rigorously eliminated in neoclassical transport calculations $[9]$.

To calculate the Ohmic contribution, the model from [6] was used. This time dependent model is described by the equation

$$
\frac{\partial E}{\partial t}=\frac{1}{\sigma}\left[\frac{\nabla^{2} E}{\mu_{0}}-E \frac{\partial \sigma}{\partial t}-\frac{\partial j_{a u x}}{\partial t}\right]
$$

and required a starting assumption for the electric field, $E$; the loop voltage measured at the vessel wall was taken to be equal at all places during the steady state time before an ELM. The only auxiliary current, $j_{a u x}$, was taken to be the bootstrap current; as this analysis was concerned only with the pedestal region (and undertaken for flux surface averaged parallel current, thus removing the Pfirsch-Schlüter currents), this is a reasonable assumption. Using the plasma geometry and experimental temperature and density profiles, a neoclassical conductivity profile, $\sigma_{n e o}$, was obtained for each time point according to the Spitzer resistivity:

$$
\sigma_{\text {Spitz }}=1.9012 \cdot 10^{4} \frac{T_{e}^{3 / 2}}{Z_{e f f} N(Z) \ln \Lambda_{e}}
$$

where $N(Z)=0.58+0.74 /(0.76+Z)$ and $\Lambda_{e}$ is the Coulomb logarithm, which was then modified as described in [10] via equations 13a-13b therein. This allowed an Ohmic current density profile, $\left\langle\boldsymbol{j}_{\mathbf{O h m i c}} \cdot \boldsymbol{B}\right\rangle$ to be derived via

$$
\left\langle\boldsymbol{j}_{\text {Ohmic }} \cdot \boldsymbol{B}\right\rangle=\sigma_{\text {neo }} E{\frac{\langle\boldsymbol{B}\rangle^{2}}{B}}^{2}
$$

A comparison between $\langle\boldsymbol{j} \cdot \boldsymbol{B}\rangle$ from CLISTE, $\left\langle\boldsymbol{j}_{\text {neo }} \cdot \boldsymbol{B}\right\rangle$, and $\left\langle\boldsymbol{j}_{\text {boot }} \cdot \boldsymbol{B}\right\rangle$ is shown in figure 7. The red line shown is the CLISTE output $\langle\boldsymbol{j} \cdot \boldsymbol{B}\rangle$ with $1 \sigma$ confidence bands which were calculated based on the technique described in section 3.1 and have similar dependences on the input parameters. The blue line shows the calculated $\left\langle\boldsymbol{j}_{\text {boot }} \cdot \boldsymbol{B}\right\rangle$ and the black line is $\left\langle\boldsymbol{j}_{\boldsymbol{n} \text { eo }} \cdot \boldsymbol{B}\right\rangle$, both with associated uncertainties. These uncertainties were calculated via a partial differentiation error propagation method, starting from the uncertainties in the fitted electron temperature and density profiles from the IDA 


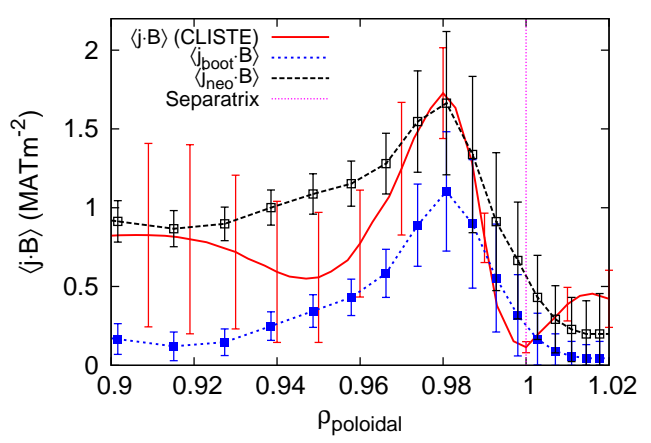

Figure 7. (Colour online) Profile of $\langle\boldsymbol{j} \cdot \boldsymbol{B}\rangle$ taken at $t_{E L M}=-3 \mathrm{~ms}$, as for the profiles above. The red line marks the CLISTE output and $1 \sigma$ confidence bands, the blue is $\left\langle\boldsymbol{j}_{\text {boot }} \cdot \boldsymbol{B}\right\rangle$, and the black shows $\left\langle\boldsymbol{j}_{\text {neo }} \cdot \boldsymbol{B}\right\rangle=\left\langle\boldsymbol{j}_{\text {boot }} \cdot \boldsymbol{B}\right\rangle+\left\langle\boldsymbol{j}_{\text {ohmic }} \cdot \boldsymbol{B}\right\rangle$, both with uncertainties derived from the electron temperature and density profiles.

routine and propagating to uncertainties in the collisionalities, bootstrap forefactors, and, finally, the bootstrap current itself. The relative uncertainties in the data are similar for all time points in the ELM cycle, apart from at the ELM crash when they increase significantly. As such, the relative uncertainty in all further plots can be understood to have a similar value as those shown in figure 7 . All profiles were taken $3 \mathrm{~ms}$ prior to the ELM crash. The excellent agreement between $\left\langle\boldsymbol{j}_{\text {neo }} \cdot \boldsymbol{B}\right\rangle$ and the CLISTE result demonstrates the accuracy of the neoclassical prediction, to within the confidence bands from both approaches.

Figure 8(a) shows the time evolution over an ELM cycle of the calculated toroidal electric field at the position of the maximum edge bootstrap current density. The increase at the ELM is predicted from Faraday's Law in order to conserve the total flux in the plasma, and decays away according to the pedestal resistive time calculated at the end of section 3. The magnitude of the variation in the electric field does leave open the possibility that a varying electric field in the pedestal causes a slow increase in the edge current which could be within the confidence bands of the local current density shown in section 3. More analysis is required to determine the relative importance of the local current density at the LFS and the flux surface averaged value in the timing of the ELM crash.

The red line shown in figure $8(\mathrm{~b})$ is the time evolution of the peak $\langle\boldsymbol{j} \cdot \boldsymbol{B}\rangle$ as output from CLISTE, with associated $1 \sigma$ confidence bands. The blue line represents the calculated $\left\langle\boldsymbol{j}_{\boldsymbol{b o o t}} \cdot \boldsymbol{B}\right\rangle$ value, and the black shows $\left\langle\boldsymbol{j}_{\text {neo }} \cdot \boldsymbol{B}\right\rangle$, as in figure 7 . The overall trend agrees with that seen in figure 4 , in that the current density drops sharply at the onset time of the ELM and slowly recovers. There is a delay seen in the recovery of $\left\langle\boldsymbol{j}_{\text {neo }} \cdot \boldsymbol{B}\right\rangle$ compared to the CLISTE output; it is possible that this is due to $\nabla T_{i}$ being taken as equal to $\nabla T_{e}$. The $T_{i}$ data at the time of this discharge was not of a sufficient temporal resolution to be used in an ELM synchronised fashion. It is also possible that the discrepancy is due to a neglected orbit squeezing modification to the 


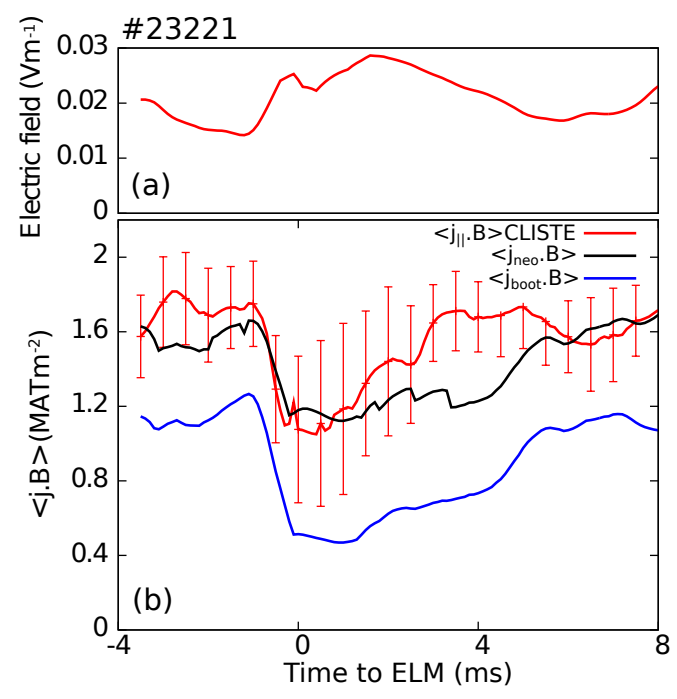

Figure 8. (Colour online) Electric field calculated at the bootstrap peak position using a resistive current propagation model (a). Peak edge values of $\langle\boldsymbol{j} \cdot \boldsymbol{B}\rangle$ from CLISTE (red), $\left\langle\boldsymbol{j}_{\text {boot }} \cdot \boldsymbol{B}\right\rangle$ (blue) and $\left\langle\boldsymbol{j}_{\text {neo }} \cdot \boldsymbol{B}\right\rangle$ (black) (b).

neoclassical resistivity model used here. It is hoped to undertake these analyses in the coming campaign with the newly upgraded diagnostics allowing high resolution $T_{i}$ measurements as well as $E_{r}$.

Two things are important to note in this analysis: (i) the current density found by CLISTE has not been constrained by $\left\langle\boldsymbol{j}_{\boldsymbol{n} \text { eo }} \cdot \boldsymbol{B}\right\rangle$ and is therefore independent of it; and (ii) while all the input profiles have been smoothed in time, each equilibrium reconstruction is independent; Faraday's law has not been taken into account, meaning the reconstructions are temporally localised, thus yielding a large number of independent comparisons.

\section{Fuelling study}

Following on from the work of Burckhart et al.[6], the same discharges were analysed for a fuelling study. Using the methodology outlined above, additional series of CLISTE reconstructions were carried out for ASDEX Upgrade discharges \#23225 and \#23226. The same broad parameters were used in all discharges, $\left(\mathrm{B}_{0}=-2.5 \mathrm{~T}, \mathrm{I}_{p}=1 \mathrm{MA}\right.$, $\mathrm{P}_{N B I}=7.5 \mathrm{MW}$ ), though the ECRH heating power was varied over the discharges. Feed forward fuelling for these two discharges was $9 \times 10^{21} \mathrm{~s}^{-1}$ and $0 \mathrm{~s}^{-1}$ respectively. Due to the variation in fuelling, the pedestal top collisionality varied in the three discharges, as shown in table 1. During the ELM cycle and across the whole pedestal the variation is larger. Table 1 also shows the comparison between the fuelling rates, collisionalities and resulting ELM frequencies.

The low field side local current density profiles from all three cases $3.5 \mathrm{~ms}$ prior to the ELM crash are shown in figure 9. It can clearly be seen that there is a difference in both the value, and, importantly, the location of the peaks, which can be explained 


\begin{tabular}{c|c|c|c} 
Discharge \# & Fuelling $\left(\mathrm{s}^{-1}\right)$ & $\nu^{*}$ range & $f_{E L M}(\mathrm{~Hz})$ \\
\hline 23226 & 0 & $0.8-2.8$ & $104 \pm 36$ \\
23221 & $5 \times 10^{21}$ & $1.5-4.2$ & $125 \pm 24$ \\
23225 & $9 \times 10^{21}$ & $3.5-6.0$ & $80 \pm 17$
\end{tabular}

Table 1. Parameters used in the fuelling study; fuelling rate, collisionality variance (calculated using the formula provided by Sauter et al.[10, 11]), and resulting ELM frequency.

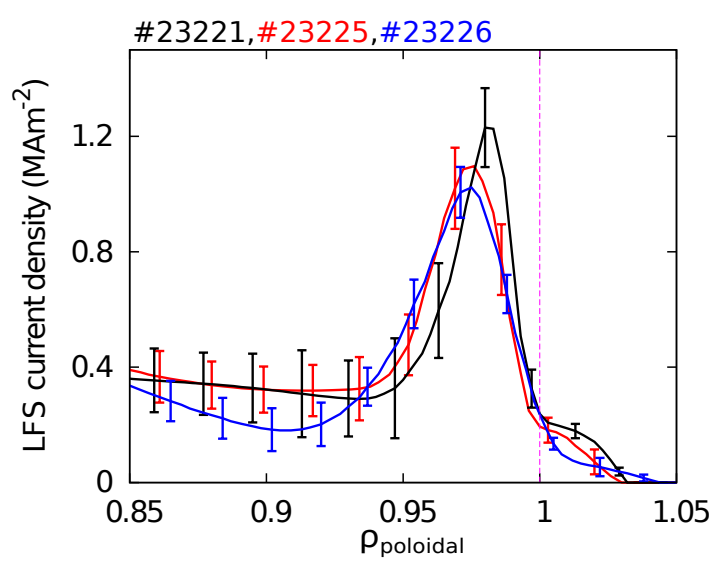

Figure 9. Pre-ELM low field side edge current density profiles for discharges \#23226, \#23221, \#23225. Each discharge exhibits a significant localised edge current density peak.

by comparing the locations of the peak edge electron density and temperature drives from [5] (figure 5); in discharge \#23225(\#23226) the $T_{e}\left(n_{e}\right)$ profile has a lower value at $\rho_{\text {poloidal }}=0.99$, due to the higher (lower) fuelling at the edge. The implication of this is that one gradient term peaks further inside, thus driving the peak current further in, while discharge \#23221 has a fuelling/heating balance which creates gradients, and hence currents, closer towards the separatrix.

If we now compare the time evolution of the peak edge current density for the three discharges (see figure 10), we again see differences. The total recovery time varies between the discharges, which also has implications for the ELM frequency due to the growth of the current density dependent peeling mode. It should be noted here that the end of each ELM cycle (i.e. beyond $10 \mathrm{~ms}$ after the ELM crash) is typically populated by only a few data points, consistent with the figures in table 1. \#23226 and \#23221 (no and low fuelling) reach saturation level within $5 \mathrm{~ms}$ of the ELM crash, while \#23225 takes longer and does not fully recover until approximately $10 \mathrm{~ms}$ after the ELM crash. The slow current density recovery of \#23225 is very similar to that of the electron pressure gradient reported for the same discharge in [6] where it was shown that the slow recovery of the temperature gradient limits the total recovery rate, though in all 


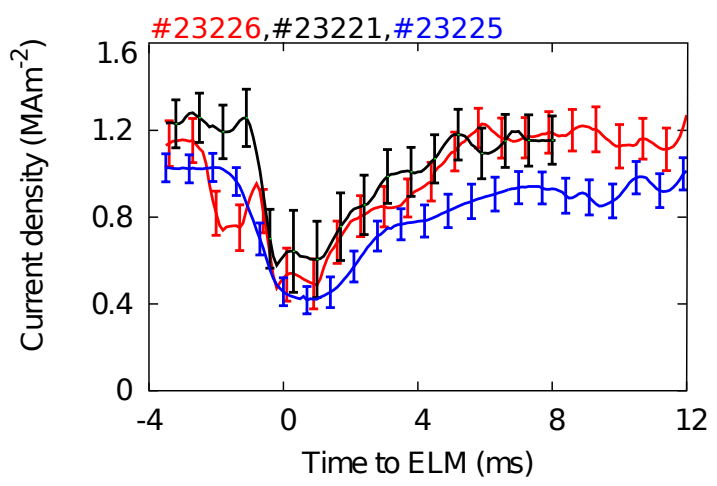

Figure 10. ELM synchronised time trace of the peak low field side local current density for discharges \#23226, \#23221, \#23225. The recovery behaviour is different for the three cases.

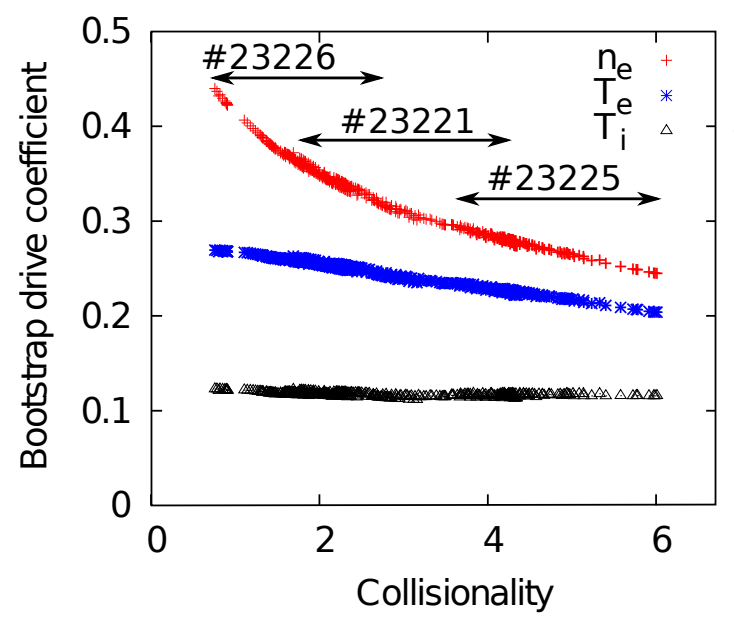

Figure 11. Coefficients for $n_{e}, T_{e}$ and $T_{i}$ drives, calculated over the large range of collisionalities presented in these three discharges. The approximate range of collisionalities over the ELM cycle for each of the three discharges is indicated. A clear drop in the density gradient term can be seen at higher collisionalities. This is also seen, though to a lesser extent, for the temperature gradient term.

three cases the reported electron pressure gradients are the same.

Because of the dependence of the $j_{\text {boot }}$ forefactors $\left(\mathcal{L}_{31}, \mathcal{L}_{32}, \mathcal{L}_{34}\right.$ in equation 1$)$ on collisionality, it is expected that increasing the density should have a large impact on the total bootstrap current. The value of the density and temperature gradient forefactors as a function of collisionality is shown for each timepoint in the three discharges in figure 11. The collisionality range for each of the discharges is indicated by arrows, and the separate forefactors are colour coded. There is a $\approx 30 \%$ drop in the density forefactor at higher collisionalities, meaning a drop in the efficiency of the electron density drive; in order to maintain the same overall current drive, a larger density gradient must be present. 


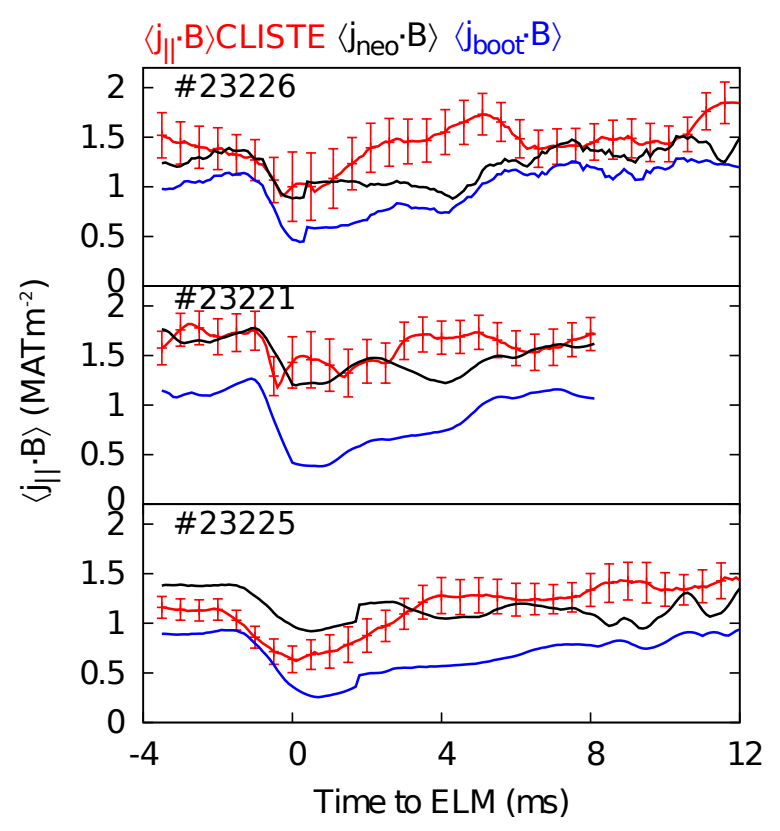

Figure 12. Edge peak values of $\langle\boldsymbol{j} \cdot \boldsymbol{B}\rangle$ from CLISTE (red), $\left\langle\boldsymbol{j}_{\text {boot }} \cdot \boldsymbol{B}\right\rangle$ (blue) and $\left\langle\boldsymbol{j}_{\text {neo }} \cdot \boldsymbol{B}\right\rangle$ (black) for discharges \#23226, \#23221, and \#23225 (top to bottom)

A comparison between the CLISTE total flux surface averaged current densities for the three cases is presented as the red lines in figure 12. Similar to the local LFS current density shown in figure 10, discharge \#23221 has the shortest recovery time and \#23225 the longest. Discharge \#23226 has a steady initial recovery rate, which then stagnates and finally fully recovers after around $10 \mathrm{~ms}$. These differences are reflected in the ELM frequencies from table 1, where discharge \#23221 has the highest frequency and \#23225 the lowest. The agreement between $\left\langle\boldsymbol{j}_{\boldsymbol{n} \text { eo }} \cdot \boldsymbol{B}\right\rangle$ (black lines) and $\langle\boldsymbol{j} \cdot \boldsymbol{B}\rangle$ from CLISTE (red lines) can also be seen in figure 12. The calculated bootstrap current is also shown in blue for reference. The discrepancy in recovery rates of the interpreted and neoclassical current densities also persists here. The impact of the $T_{i}=T_{e}$ constraint is further highlighted by the low collisionality case of discharge \#23226, while only a small difference in the recovery rates of the high collisionality discharge \#23225 can be seen. As a side note on the interpretation of the magnitude of $\langle\boldsymbol{j} \cdot \boldsymbol{B}\rangle$ from CLISTE in the pre-ELM timerange, it should be noted that the confidence bands are calculated based on the relative error of the local current density profiles and are thus also dependent on the magnitude of the flux surface averaged current density. If the absolute magnitude of the confidence bands from the recovered phase of the ELM cycle were applied here, the CLISTE and theory timetraces would agree to within these confidence bands.

The contrasting recovery histories of the individual contributions to the bootstrap current for the three discharges are plotted in figure 13. Corresponding to the slow recovery of the electron temperature gradient reported for discharge \#23225 in [6], the electron temperature drive of the bootstrap current also recovers slowly, as can be seen 


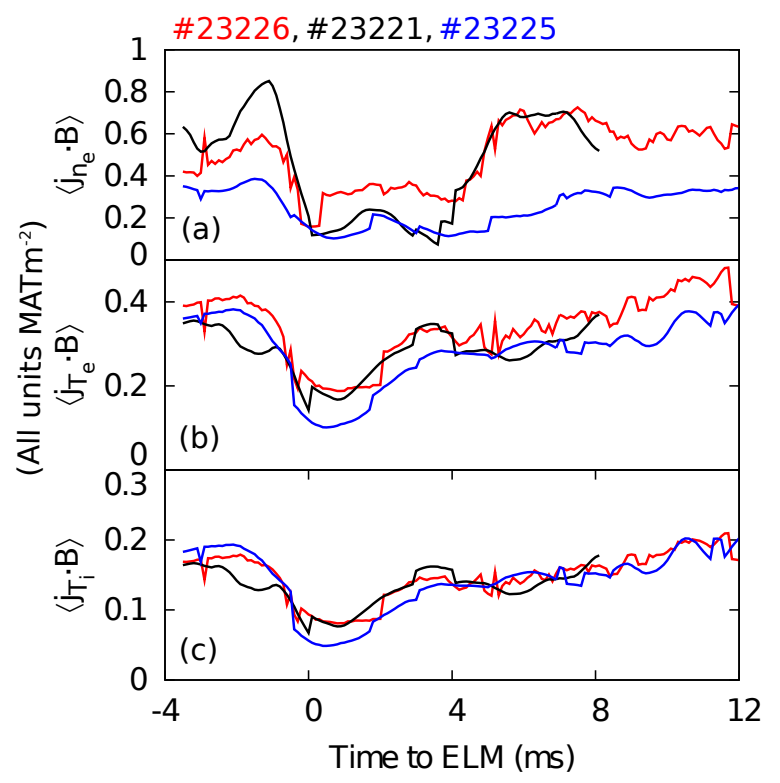

Figure 13. Comparison of the three bootstrap component drives for discharges \#23226, \#23221, \#23225. (a) $n_{e}$ drive, showing a clear drop for the highly fuelled case of \#23225, (b) $T_{e}$ drive, which remains more or less constant, though recovery rates differ, (c) $T_{i}$ drive, which contributes only a small portion to the total drive.

in figure 13(b). According to the forefactors shown in figure 11, the electron density gradient term becomes less efficient in the cases with higher fuelling. However, since the collisionality was increased through fuelling, there is a compensation for the lower drive efficiency for discharge \#23221. Discharge \#23225 on the other hand, which has the highest fuelling rate, does not see this compensation, and so the electron density gradient driven current drops significantly to the order of the electron temperature gradient term. Although the electron temperature gradient efficiency also drops with increasing collisionality, the current density driven by this term decreases only slightly in discharge \#23225. The ion temperature gradient term typically forms a small fraction of the total bootstrap current, though may play a more significant role in overall recovery if its recovery rate were to differ from the electron temperature gradient.

In the cases of these discharges, the ELM frequency follows the same trend of the density gradient contribution to the bootstrap current drive. It is acknowledged that this is, however, a very small sample size and much further study is needed in this area before any firm conclusions can be drawn. This fuelling study provides valuable information on the effects of fuelling on the bootstrap current, and its possible effects on the ELM cycle. 


\section{Conclusions}

The results presented here show the first ELM resolved current density measurements at ASDEX Upgrade. A high temporal resolution has been obtained due to the use of an ELM-synchronisation technique, allowing both the local and flux-surface-averaged current densities to be determined on a sub millisecond timescale. A strong edge peak in the current density was seen, as expected from the steep pressure gradient in this region. Several other equilibrium quantities can also be analysed on this timescale, notably the movement of the plasma separatrix. The reduction of the plasma radius is strongly indicative of plasma being moved outside the last closed flux surface, allowing the decrease of edge current density to proceed faster than would be expected from resistive timescales. This rapid loss causes an inductive response from the plasma which is accompanied by an increased SOL current, leading to a total increase in the plasma current.

The sharply peaked edge current density is also seen in the flux surface averaged value. This reflects only the Ohmic and bootstrap drives of the current (since the Pfirsch-Schlüter is rigorously zero in this formulation), which allows comparison with theoretical calculations. These calculations, using formulae derived from neoclassical theory, were shown to be in good agreement with the results obtained from analysis using the CLISTE code, providing strong evidence that the expressions for calculating neoclassical currents provided by Sauter et al. [10,11] are quantitatively correct in the pedestal region.

Three discharges were then analysed as part of a fuelling study. The LFS local edge current density profiles vary in both width, location, and height of the peaks. The current density recovery times for each of the discharges also varied in accordance with the pressure gradient recovery times reported in [6]. Neoclassical calculations of the edge current density in three discharges were also made. It was seen that the density contribution to the total bootstrap current drops significantly at higher fuelling rates, due mainly to the lower efficiency of this drive in the high collisionality regime. This is compensated in part by the increased density gradient in the edge. A suppression of the density gradient driven bootstrap current was seen for discharge \#23225 and was also accompanied by a significantly longer recovery time of the electron temperature gradient driven bootstrap current. No firm conclusions on the relationship between ELM frequency and fuelling rate can be drawn from these results, though there is an indication that the density gradient contribution to the current density could be an important factor.

\section{Acknowledgements}

The authors would like to thank Prof. K. Lackner for valuable discussions. This work was supported by EURATOM. 


\section{References}

[1] H R Wilson, S C Cowley, A Kirk, and P B Snyder. Plasma Physics and Controlled Fusion, 48(5A):A71-A84, May 2006.

[2] H Zohm. Plasma Physics and Controlled Fusion, 38:105-128, March 1996.

[3] G Federici, A Loarte, and G Strohmayer. Plasma Physics and Controlled Fusion, 45(9):1523-1547, September 2003.

[4] A Loarte et al. Plasma Physics and Controlled Fusion, 45:1549-1569, 2003.

[5] E Wolfrum, A Burckhart, R Fischer, N Hicks, C Konz, B Kurzan, B Langer, T Pütterich, H Zohm, and ASDEX Upgrade Team. Plasma Physics and Controlled Fusion, 51(12):124057, December 2009 .

[6] A Burckhart, E Wolfrum, R Fischer, K Lackner, H Zohm, and ASDEX Upgrade Team. Plasma Physics and Controlled Fusion, 52(10):105010, October 2010.

[7] M F M De Bock, J Citrin, S Saarelma, D Temple, N J Conway, A Kirk, H Meyer, and C A Michael. Plasma Physics and Controlled Fusion, 54(2):025001, February 2012.

[8] D. M. Thomas, A. W. Leonard, R. J. Groebner, T. H. Osborne, T. A. Casper, P. B. Snyder, and L. L. Lao. Physics of Plasmas, 12(5):056123, 2005.

[9] M. Coronado and H. Wobig. Physics of Fluids B: Plasma Physics, 4(5):1294, 1992.

[10] O. Sauter, C. Angioni, and Y. R. Lin-Liu. Physics of Plasmas, 6(7):2834, 1999.

[11] O. Sauter, C. Angioni, and Y. R. Lin-Liu. Physics of Plasmas, 9(12):5140, 2002.

[12] M. Wade, M. Murakami, and P. Politzer. Physical Review Letters, 92(23):1-4, June 2004.

[13] D J Kelliher, N C Hawkes, P J Mc Carthy, and Jet EFDA Contributors. Plasma Physics and Controlled Fusion, 47(9):1459-1473, September 2005.

[14] P.J. McCarthy. Physics of Plasmas, 6(9):3554, 1999.

[15] P.J. McCarthy and ASDEX Upgrade Team. Plasma Physics and Controlled Fusion, 54(1):015010, January 2012.

[16] A. Kallenbach et al. Nuclear Fusion, 48(8):085008, August 2008.

[17] R Fischer, C J Fuchs, B Kurzan, W Suttrop, E Wolfrum, and ASDEX Upgrade Team. Fusion Science and Technology, 58(2):675-684, 2010.

[18] N. A. Salmon. International Journal of Infrared and Millimeter Waves, 15(1):53-60, January 1994.

[19] E. Wolfrum, F. Aumayr, D. Wutte, Hp. Winter, E. Hintz, D. Rusbuldt, and R. P. Schorn. Review of Scientific Instruments, 64(8):2285, 1993.

[20] R Fischer, E Wolfrum, J Schweinzer, and ASDEX Upgrade Team. Plasma Physics and Controlled Fusion, 50(8):085009, August 2008.

[21] O. Gehre. International Journal of Infrared and Millimeter Waves, 5(3):369-379, March 1984.

[22] G.G. Lister. FAFNER - A fully 3-D neutral beam injection code using Monte Carlo methods. IPP Report, (4-222), 1985.

[23] P.J. McCarthy. Inverse Problems, 19:643-663, 2003. 